Estudios Románicos, Volumen 28, 2019, pp. 65-76

ISSN: 0210-491

eISSN: 1989-614X

DOI: https://doi.org/10.6018/ER/381061

\title{
LA RELACIÓN EPISTOLAR DE CLAUDIO MAGRIS Y BIAGIO MARIN
}

(The epistolar relationship between Claudio Magris and Biagio Marin)

\author{
Encarna Esteban Bernabé* \\ Universidad de Murcia
}

\begin{abstract}
In 2014, Garzanti publishes all the existing letters exchanged for 27 years by the Germanist and writer Claudio Magris and the poet Biagio Marin. The epistolary literature is a genre very close to psychology or even to philosophy, since in the written communication that is established between the interlocutors usually appears the deepest ego of each one of those involved. This is the case of the work that concerns us, $\mathrm{Ti}$ devo tanto di ciò che sono. Carteggio Magris-Marin. In these letters, the two great writers discuss philosophy, literature, theology, history and politics, while exchanging news and anecdotes of their own lives. Both the scholar and the curious reader will find in this work keys of understanding of the work of these two great men of the Italian letters.
\end{abstract}

Keywords: Claudio Magris; Biagio Marin; Letters.

Resumen: En 2014 la editorial Garzanti publica la totalidad de las cartas existentes que durante veintisiete años intercambiaron el germanista y escritor Claudio Magris y el poeta Biagio Marin. La literatura epistolar es un género muy cercano a la psicología o incluso a la filosofía, ya que en la comunicación escrita que se establece entre los interlocutores suele aparecer el yo más profundo de cada uno de los implicados. Este es el caso de la obra que nos ocupa, Ti devo tanto di ciò che sono. Carteggio Magris-Marin. En esas cartas, los dos grandes literatos discuten de filosofía, literatura, teología, historia y política, al tiempo que intercambian noticias y anécdotas de la propia vida. Tanto el estudioso como el lector curioso encontrará en este trabajo claves de comprensión de la obra de estos dos grandes hombres de las letras italianas.

Palabras clave: Claudio Magris; Biagio Marin; Cartas.

* Dirección para correspondencia: Encarna Esteban Bernabé. Dpto. Filología Francesa, Románica Italiana y Árabe. Facultad de Letras. Campus La Merced. C/Santo Cristo, no 1, 30001, Murcia. (encarna.esteban@um.es) 


\section{Introducción}

Claudio Magris ha confesado en distintas ocasiones que le debe mucho de lo que hoy es a su maestro y amigo Biagio Marin (1891-1985), el poeta de Grado, un pequeño municipio de Gorizia, de la región del norte de Italia, Friul-Venecia Julia. En 2014 Renzo Sanson publica en Garzanti las cartas que durante más de treinta años se intercambiaron los dos grandes intelectuales italianos. El título elegido para la colección epistolar es exactamente esa confesión de Magris de deuda vital, moral y espiritual con el poeta: Ti devo tanto di ciò che sono. Carteggio con Biagio Marin (Magris; Marin 2014). Un año antes de esta publicación, en 2013, la revista de cultura y literatura Il Giannone consagra un monográfico a la figura de Magris (Motta 2013). En este número especial, Renzo Sanson publica un extenso artículo sobre la existencia de esas cartas ricas de vida y literatura, que son hoy un legado importantísimo para los estudiosos, tanto de Magris, como del poeta de Grado, Biagio Marin. En dicho artículo se recogían diecisiete de esas cartas como anticipo de la publicación de Garzanti, que reúne la totalidad de las misivas que se conservan actualmente. Aún no contamos con una traducción al castellano de esta obra, pero la belleza de sus palabras y la profundidad de las palabras que intercambiaron durante años los dos literatos, merecen que detengamos nuestra atención en ellas.

En este trabajo se pretende el acercamiento del lector al universo emocional del Premio Príncipe de Asturias de las Letras 2004, y a la influencia que produjo en él la amistad con el anciano poeta Biagio Marin, y que ha quedado plasmada en las hermosas cartas que han llegado hasta nosotros.

Si hoy podemos disfrutar de este valioso legado epistolar, lo debemos, fundamentalmente, a la mutua admiración que se profesaban los dos autores. Esta admiración los llevó a ser cautelosos guardianes de las palabras del otro, pues ambos eran sabedores en todo momento, de que esas cartas encerraban algo mucho más trascendental para las letras italianas que un simple intercambio epistolar entre dos amigos.

En un primer apartado nos detendremos en una breve presentación de los protagonistas del epistolario que nos ocupa, pero no tanto de la persona y la obra -ampliamente ya estudiadas - sino más bien, de la personalidad, del genio y, sobre todo, de lo que esa relación de amistad supuso para cada uno de ellos, en sus vidas y en sus obras. De esta manera, podremos posteriormente adentrarnos en algunas de esas bellísimas páginas que nos han dejado estos dos grandes literatos italianos.

\section{Los interlocutores}

De Claudio Magris podemos decir, sin miedo a caer en la hipérbole, que es una de las mayores figuras de la literatura universal contemporánea. El autor de Il mito asburgico (1963), Danubio (1988), Otro mar (1992), Microcosmos (1999) o El infinito viajar (2008), entre otros, es hoy un prestigiosísimo germanista, ensayista y traductor estudiado y admirado en las universidades más importantes del planeta. Sin embargo, cuando se produce ese primer encuentro con el poeta Marin, Magris no es más que un 
joven de dieciséis años, estudiante de Secundaria en el instituto Dante de Trieste. Su padre, Duilio Magris, que trabajaba en Assicurazioni Generali, es quien los presenta en 1955. Biagio Marin, de sesenta y tres años de edad y cercano ya a la jubilación, era el encargado de la biblioteca de la sede central de Trieste.

Marin ya era un reconocido poeta italiano y había recibido el premio de poesía Barbarani en 1952 por su obra I canti dell'isola, hecho que supuso un punto de inflexión en la vida y la carrera del poeta. Este premio despertó la atención de la crítica italiana del momento, especialmente de Carlo Bo y Pier Paolo Pasolini, cuya relación también quedó plasmada en distintas cartas. El padre de Magris y Marin mantenían una estrecha amistad desde hacía unos años. El joven Magris no olvidará nunca ese primer encuentro que lo transformó profundamente tras un auténtico "flechazo", como él mismo confiesa: "Ricordo benissimo quel giorno alle Generali. Mio padre aveva grande ammirazione per Marin che a sua volta aveva molta stima e affetto per lui. E così gli aveva parlato di me. Io sono andato a conoscerlo ed è stato subito un colpo di fulmine." (Sanson 2013: 29).

También Marin quedó impresionado de la inteligencia del joven Magris. En ese primer encuentro la conversación fluyó entre los dos, como si la diferencia de edad no fuese tal y en realidad se tratase de dos viejos amigos que discuten asiduamente de filosofía y literatura. Los diálogos socráticos de Platón, Goethe y los Evangelios ocuparon la mayor parte del diálogo de ese primer encuentro en la biblioteca, al que siguieron muchos más.

El anciano poeta, marcado por la dramática pérdida de su hijo Falco, víctima mortal de la guerra en 1943, vio en Magris desde un primer momento, un nuevo hijo, un nuevo regalo que la vida le concedía para devolverle la ilusión y la esperanza, que creía ya perdidas. Son numerosas las referencias que encontramos en las cartas en las que Magris es el "figliolo d'anima". El poeta vio en este despierto muchacho un futuro brillante y preconizó, desde ese temprano encuentro, el éxito del autor de Danubio. En su papel de "padre" lo acompañó toda la vida; lo ayudó en las decisiones importantes, tanto profesionales como personales, e incluso lo reprendió en no pocas ocasiones. Pero la admiración que sentía hacia él, hacia su vasta cultura, su inteligencia fuera de lo ordinario y su maravillosa prosa fue lo que selló una unión filial con el escritor más allá de los lazos reales de sangre. Además, Biagio Marin eligió a Claudio Magris como su "Juan Bautista", el heredero de su obra poética; el "apóstol" que abriría las puertas de la gloria de la que aún no disfrutaba plenamente. La poesía de Marin, de indiscutible sensibilidad y calidad formal, encontró la indiferencia del gran público durante la vida del poeta, especialmente debido a su elección del triestino y el dialecto de Grado como lengua de expresión poética, el graisan, un dialecto de matriz veneciana; elección que le supuso la injusta etiqueta de poeta provincial por muchos críticos literarios de la época. El propio Marin es consciente de la dificultad que entraña la lectura de sus versos y así lo expresa a Magris en una carta fechada en Grado, el 10 de marzo de 1974: "Io lo so, che la particolarità del mio linguaggio e del mio mondo sono ostacolo alla facile lettura, a quella spontanea immedesimazione che io ritengo necessaria premessa al valido giudizio estético.” (Magris; Marin 2014: 301) 
Sin embargo, bien distinta es la opinión de la estudiosa de la obra mariniana, Edda Serra. Serra afirma que la grandeza de Marin está en el hecho de haber traducido en poesía, el mundo de la región Friul-Venecia Julia, y haber hecho de esta Región y especialmente de Grado, un símbolo existencial para toda la humanidad:

Né Marin è mai provinciale, neanche quando canta il piccolo mondo locale. La sua infatti è vocazione alla unità, alla sintesi, ed al superamento. Di istinto egli si proietta in una dimensione unitaria più ampia: all'interno della regione fondendo voci dissonanti, invitando al superamento del particolare; del resto proiettando la sua esperienza negli spazi più vasti, nazionali, ed oltre. [...] La grandezza di Marin è di avere tradotto in poesia il mondo regionale del Friuli-Venezia Giulia, Grado innanzitutto, facendolo diventare simbolo esistenziale non più solo nostro, con i mezzi offerti da quel mondo: il dialetto, le immagini, i valori, i temi, le contraddizioni.

La sua è però voce alta di civiltà italiana nella cui storia ha lasciato un'impronta viva ed ampia nel suo commisurarsi con la realtà di confine nello spazio più ampio dell'Europa della fine del secolo scorso e di questo secolo, di cui pure si nutre. (Serra 1992: 101)

De la misma opinión es el crítico literario Pier Vincenzo Mengaldo, que en su antología de poetas italianos del Novecento incluye a Biagio Marin, comparando su poética con la de los españoles Antonio Machado y Juan Ramón Jiménez o el italiano Umberto Saba (Mengaldo 1978: 502).

La profecía de Marín se vio cumplida. Claudio Magris habría de convertirse en el embajador de la poesía del amado "padre espiritual". A lo largo de su dilatada carrera, Magris ha hablado de Marin como un segundo padre, cuya huella ha marcado profundamente su vida y su pluma. Son muchos los congresos y jornadas de estudio sobre la obra mariniana que han sido promovidos por él, dentro y fuera de Italia. Además, cumplió el sueño del poeta al ocuparse de la introducción de su primera antología Nel silenzio più teso (1980) y de la selección y las notas de la antología publicada por Garzanti (1991). Las numerosas referencias a la grandeza de la poesía de Marin en los artículos y ensayos de Magris han despertado la curiosidad de lectores y estudiosos en todo el mundo. Recordemos que Danubio, obra traducida a más de veinte idiomas, se cierra con una cita de Biagio Marin:

Pero el canal corre leve, tranquilo y seguro hacia el mar, ya no es canal, límite, «Regulation», sino flujo que se abre y se abandona a las aguas y a los océanos de todo el globo, y a las criaturas de sus profundidades. «Fa che la morte mia, Signor -dice un verso de Marin - la sia comò 'l scôre de un fiume in t'el mar grando.» (Magris 1997: 370)

\section{La relación epistolar}

A la pregunta de Renzo Sanson sobre la importancia que la relación con Marin ha dejado en Magris, este responde que el auténtico protagonista de esta relación es la 
relación en sí; ni Magris ni Marin. Magris habla de un tercer protagonista que es el encuentro entre los dos. En ese espacio de encuentro, cada uno de los interlocutores sufre una transformación, una mutación sutil, pero duradera, que hará que ya nunca vuelvan a ser los mismos de antes. Marín solía valerse del lenguaje religioso para expresar esta idea del tercer protagonista y decía que era como la intuición del Espíritu Santo, cuya razón de ser es precisamente esa, la relación de amor entre el Padre y el Hijo. Magris recuerda esta imagen del amigo para hablar de la relación epistolar que mantuvieron hasta la muerte del poeta:

Forse nessuno come Marin mi ha fatto sentire come, nel rapporto fra due persone, il vero protagonista non sia l'uno o l'altro degli interlocutori, bensì il loro rapporto, quel "terzo" che è il loro incontro, in cui ognuno muta, in certa misura, nel rapporto con l'altro e non è più lo stesso di prima. Marin, che amava riprendere tante espressioni del linguaggio religioso, diceva che l'intuizione dello Spririto Santo è appunto questa: la realtà di quel "terzo" che è il rapporto di per sé, in particolare quando si tratta di un rapporto d'amore, nel più ampio senso della parola. (Sanson [Magris; Marin 2014]: 373)

La relación epistolar empezó cuando el joven Magris salió de su Trieste natal para cursar los estudios de Lengua y Literatura Extranjeras en la Universidad de Turín. El brillante estudiante, a pesar de contar con el apoyo y el amor de su padre biológico, sintió la necesidad de "ser adoptado" por Marin como hijo espiritual. Para él, el intercambio dialéctico con el poeta se convierte en una especie de gimnasio intelectual que contribuye a formar y reforzar su carácter (Sanson [Magris; Marin 2014]: 14). Dicho diálogo resistió los envites del tiempo y sólo se interrumpió con la muerte de Biagio Marin en 1985. Marin compartió con el joven Magris los avatares de los años de la Universidad; lo animó a la publicación de su tesis doctoral; fue testigo de su gran amor, Marisa Madieri; del nacimiento de sus dos hijos, Francesco y Paolo, de los éxitos profesionales y literarios, de sus viajes por el mundo, de sus alegrías y de sus inseguridades. Encontramos en estas cartas confesiones de dolor y miedos de ambos escritores. Marin comparte con su joven amigo el dolor que ha provocado en él la trágica muerte de su yerno. Del mismo modo, Magris comparte la soledad que siente en la Universidad de Turín o el desgarro de la pérdida de su tía, hecho que abre una de las reflexiones más profundas del poeta en la carta de respuesta fechada el 27 de enero de 1960:

Nessuna esperienza arricchisce una vita quanto la morte di una persona cara. [...] L'eredità della zia, della giovane donna stroncata, potrà fluire in te, alargando il tuo orizzonte e potenziando la tua giovane forza. Forse ora si accenderà tra tu e lei il dialogo veramente fecondo. Te lo auguro. (Magris; Marin 2014: 102)

Magris necesita la voz de ese "padre" que lo sostiene y que arroja luz a sus dudas existenciales, de manera especial tras la repentina muerte de su padre biológico, y así se lo hace saber en la carta del día 28 de febrero de 1971: 
Vorrei che tu sentissi, che tu capissi quanto tu sei importante, fondamentale nella mia vita: sei l'unico padre che io ancora abbia (e ho tanto bisogno di padri, di guide, di maestri!), l'unico cui possa attingere forza, verità, salute.

Credo che a nessuno tu abbia dato come a me: il sapere che tu ci sei, che posso correre da te, mi dà fiducia, serenità. Tu mi dai ciò che forse tu stesso da giovane non hai avuto: l'equilibrio, l'armonia. Mamma e Marisa ti benedicono per il sostegno che rappresenti per me: ogni volta mi restituisci a loro più sicuro e sereno. Sono io che ho bisogno di te, talora con l'amoroso egoismo dei figli verso i genitori, ma questo ti fa capire quanto tu sia vivo. (Sanson 2014: 270-271)

Sin embargo, a medida que pasan los años, los ritmos vitales de nuestros protagonistas se distancian. Para Marin las horas transcurren lentamente en su Grado natal. Necesita las cartas de Magris para encontrar el aliento que le empieza a faltar. En él ha puesto sus esperanzas, el sueño de verse reconocido en esa Italia que, a su juicio, no ha sabido valorar su obra. Pero Magris no dispone del tiempo material que su amigo le reclama. Él se divide entre la incipiente carrera, la familia, los viajes y las publicaciones. Sus cartas son en ocasiones unas simples líneas para no caer en la descortesía del silencio absoluto. Ya no hay tiempo para los profundos debates que entablaron en el periodo universitario del escritor, y que tanta vida proporcionaron al anciano poeta. En cualquier caso, los reproches por parte de Marin fueron casi una constante desde el inicio de la relación epistolar. El poeta solicita una mayor presencia, personal y escrita desde el principio, como podemos comprobar en la carta fechada el 30 de mayo de 1959: "Caro Claudio, si vede che sei stanco perfino della scrittura, mi prometti poi, come già altre volte, di venirmi a vedere. Ma quando sei qua, il tuo ambiente nativo e sociale ti riprende ancora tanto, che neanche allora hai veramente tempo da perdere con me e con te" (Magris; Marin 2014: 98).

Incluso cuando el frenesí del joven se refleja en una caligrafía ilegible, la llegada de cada carta se traduce en Marin en un soplo de vida: "Caro Claudio, non voglio fare a meno di ringraziarti per la tua ultima, scritta in fretta, con una calligrafia da far disperare, ma, come tutte le tue lettere, dono di vita. Grazie" (Magris; Marin 2014: 97).

Biagio Marin se alimenta de palabras, las pronunciadas en diálogo profundo, las leídas y las escritas. Se lamenta de la brevedad de las cartas de Magris en sus diarios personales y, de forma clara, se lo hace saber al joven germanista. Marin necesita dejar constancia de su vida, de sus reflexiones y ¿cómo no? de su creación poética, sobre el papel que lo trascenderá. Por ello, además de las cartas, decidió plasmar su mundo interior en su zibaldone, su diario personal. El de Marin es un diario literario, pues desde el principio, el poeta escribe esos cuadernos con intención literaria. Él sabe que llegará el día -como así ha sido- en el que Italia descubra su obra y entonces se asomarán a sus reflexiones íntimas para disfrutar plenamente de su poesía. Marín también confía en el éxito que le llegará a su querido Claudio Magris, razón por la cual transcribe la mayor parte de las cartas que recibe en su diario personal, especialmente las de la segunda etapa del epistolario, a partir de los años setenta, cuando Magris ya ha conseguido parte de ese éxito preconizado por el poeta. De esta manera, asegura su permanencia 
y las pone a disposición de futuros estudiosos de su obra. Expresa explícitamente este deseo en su diario el 31 de julio de 1963: "Perché le copio? Perché sono una ricchezza. Mi appartengono, sono momenti della mia vita. Non si potrà giudicare della mia vita, senza questi documenti della vita che solo in parte appartiene ai loro scrittori." (Sanson [Magris; Marin 2014]: 31).

El contrapunto lo encontramos en Magris, que en su frenética actividad decide en ocasiones contestar a las misivas de Marin con un medio más inmediato como es el teléfono. El autor de Microcosmos no escribe sus cartas con conciencia literaria. Simplemente, tal y como señala Sanson, son un medio de comunicación real con su amigo: "Per il giovane Magris, invece, il rapporto epistolare è strettamente confidenziale. Sostituisce la comunicazione faccia a faccia, con il vantaggio che «lo scrivere è un modo di parlare senza essere interrotti» (Renard)" (Sanson [Magris; Marin 2014]: 18).

La insistencia de Marin sobre la necesidad de un heredero es recurrente en las cartas. Desde los inicios de la relación epistolar, el poeta anuncia al joven de Trieste que ve en él ese heredero espiritual y literario que se encargará del orden y la publicación de toda su obra, incluidos los diarios y el epistolario. Así se lo hace saber en la carta fechada el 5 de diciembre de 1959: "I miei diari sono sfoghi, a volte troppo violenti, perché si possano pubblicare. Bisogna passino almeno 50 anni. E poi chi sarà che vorrà mettere gli occhi in tante pagine? E chi l'erede? Non capisci che mi occorre un figliolo prima di morire?" (Magris; Marin 2014: 99).

Las dos grandes personalidades que se dan cita en el encuentro epistolar sienten una auténtica hambre de diálogo, de debate fecundo que revista la mundanidad de la amistad de una experiencia casi mística. Esta fecundidad epistolar nos ha proporcionado un riquísimo legado literario y personal que nos invita a la reflexión sobre las grandes cuestiones trascendentales de la vida: la ética de los valores, el compromiso existencial, el catolicismo, especialmente el concepto paulino del "buen combate", la función de los intelectuales, la definición de poesía y una amplísima reflexión sobre los grandes nombres de la literatura universal. Resulta difícil recoger aquí la totalidad de los temas tratados en estas cartas, pero veamos a continuación una pequeña muestra de algunas de las cuestiones que ocuparon el debate que, durante años, acompañó a estos dos grandes nombres de las letras italianas.

\subsection{La importancia del diálogo con el otro}

En las cartas son muchas las referencias a la importancia del diálogo profundo con el prójimo. Sólo en el diálogo puede darse el auténtico encuentro que nos acercará al otro, tanto si sus ideas se encuentran en comunión con las propias, como si son opuestamente contrarias. En el estudio, en la lectura de los grandes filósofos, teólogos o literatos, encontramos las ideas, pero no al otro en sí. Para que ese encuentro trascendental se dé es imprescindible confrontarse dialécticamente. La palabra posee una fuerza sobrenatural que confiere casi una divinización a la persona. La capacidad de comunicar con el otro es lo que nos identifica como seres humanos y tanto Marin como Magris confiesan ser seres hambrientos de diálogo: 
Da ciò l'angoscia della morte che non è disperazione della validità dei valori, ma non voler scomparire. E da ciò -passando alla sfera psicologica, pratica - un po' tutto il mio stile di vita, basato tutto su persone e su volti amati che su problemi generali. Io sento di vivere, e di realizzare me stesso solo nei vincoli amorosi che mi legano a certe persone insostituibili, a cui devo molto di me e a cui cerco di regalarmi, tentando di realizzare «dialoghi». Per questo nessuna distanza intellettuale, politica eccetera mi ha mai allontanato da nessuno, se vi era viva e umana verità nel nostro rapporto. (Magris; Marin 2014: 104)

Esta confesión de Magris provoca la respuesta de Marin, quien identifica esta necesidad de amor en el diálogo con los otros con la presencia del Espíritu Santo. Esto hace que en esa carta de respuesta el poeta llame a Magris: "giovane affamato di spirito santo" (Magris; Marin 2014: 104). Es decir, de nuevo se está identificando el diálogo entre dos personas con la presencia divina, en esa especie de tercer protagonista del que ya hemos hablado.

En una carta anterior, Marin habla de la magia que se produce cuando se da el auténtico diálogo. Se trata de bajar a la profundidad del alma del interlocutor que, abandonando el simple intercambio de ideas intelectuales, se abre con humildad y amor a las palabras del otro. Marin recuerda a su joven amigo, que ese es el tipo de diálogo que se ha entablado entre ellos:

Caro Claudio, i dialoghi premettono due uomini pari; ad essi intonati a priori nel profondo dell'anima. Se no, non sono possibili. Vi ha sì lo scambio di idee scientifiche, $\mathrm{o}$, comunque, già intellettualmente prefissate, ma il dialogo che interessa noi è frutto di un miracolo di umiltà e di amore. (Magris; Marin 2014: 94)

Otra de las funciones del diálogo, especialmente del que se da en la relación epistolar, es la oportunidad de adquirir un mayor conocimiento interno. La psicología moderna insiste en la utilidad de la palabra escrita como medio para desarrollar el aforismo griego del "conócete a ti mismo", pero Marín va un poco más allá cuando afirma que esta es la única razón de ser de las cartas: "Non c'è ragione che tu mi scriva, se non senti il bisogno di chiarire te stesso nel dialogo." (Magris; Marin 2014: 109). Esta premisa se mantiene en Marin a lo largo de todo el epistolario. En una carta de 1974, cuando pasaba ya de los ochenta años, vuelve a dejar constancia de ese deseo profundo de diálogo verdadero y añora los años pasados en los que se dio, lamentándose así de la ausencia del mismo en la parte final de la relación, debido especialmente a los diferentes ritmos vitales de los dos amigos:

Forse quel dialogo al quale aspiro, e che tra noi una volta avveniva miracolosamente, fuori di ogni intenzione, di ogni consapevolezza, non è più possibile per la diversità di ritmo e di intonazione tra te nella pienezza della tua forza, nel mezzogiorno della tua intelligenza e me, nella fase del tramonto. Dove magari sono possibili intuizioni luminose e esperienze pregnanti, ma che se non espresse in forme perfette, subitamente perdono il loro valore. (Magris; Marin 2014: 310) 
Aunque son muchas más las referencias que encontramos a la importancia del diálogo, cerraremos este apartado con la cita de una de las cartas de los últimos años, en la que Marin confiesa que el simple hecho de verbalizar al amigo el propio dolor, aún a riesgo de mostrar la vulnerabilidad y fragilidad del alma, provoca un alivio sanador. Pero la condición para que esa sanación se produzca es la apertura incondicional del corazón y de la mente del interlocutor: "Perché ora scrivo a te queste cose? Prima di tutto perché traducendo il sentimento che mi tormenta in pensiero, un qualche sollievo me ne viene; poi per la speranza che tu possa accogliermi a cuore e a mente aperti." (Magris; Marin 2014: 301)

\subsection{La existencia de Dios}

Otro de los temas claves del epistolario entre Magris y Marin es la cuestión de Dios. Su existencia, su presencia en la vida del hombre del siglo XX y su relación con el ser humano ocupa una buena parte de la reflexión de los dos literatos.

La espiritualidad de Marin - y por lo tanto, en buena medida, también la de Magris - es la del Dios encarnado en sus criaturas: "lavora sereno e sicuro, perché Dio è in te, e tu sei il tempio del Signore, e espressione, manifestazione di Dio." (Magris; Marin 2014: 89). Cada hombre se presenta como una teofanía en el mundo. Este conocimiento es el que confiere la calidad de humano al hombre y en él reside toda su dignidad. Marin desarrolla este concepto en distintas cartas a lo largo de toda su vida. De ahí nace esa necesidad de interioridad agustiniana en la que tanto insiste; pero la originalidad de Marin está en el atribuir al Humanismo el mérito de haber inducido a esa interioridad, incluso sin ser consciente de ello:

Penso che anche l'Umanesimo abbia avuto la sua sincera religiosità, anche se metteva nello uomo la radice del divino. In interiore homine habitat veritas, aveva detto già Agostino, e questa immanenza non esclude la trascendenza rispetto a ogni immediatezza psicologica o naturale. (Magris; Marin 2014: 96).

El anciano poeta, cerca ya de los noventa años, insiste de nuevo en la presencia de Dios en el hombre, sin que esto suponga la negación de la inconmensurable distancia entre lo humano y lo divino, y una vez más, la referencia de autoridad es el santo de Hipona: "Mi ha insegnato Agostino che la distanza fra l'uomo e Dio è infinita, ma che ogni passo che l'uomo faccia verso Dio è già in Dio." (Magris; Marin 2014: 343)

Sin embargo, no falta en el discurso del poeta una crítica feroz, y hasta en ocasiones despiadada, a la Iglesia. Su experiencia de Dios, realmente profunda, tal y como se desprende de sus cartas y diarios íntimos, es individualista, solitaria, casi aislada y melancólica. Marin prefiere vivir su espiritualidad al margen de la propia Iglesia, aun sabiendo que esto lo deja "huérfano" en su alma, puesto que es consciente de la necesidad del encuentro con el otro para crecer en su camino espiritual. Sin embargo, esta carencia es aceptada como intrínseca a su persona. Marin no muestra ningún reparo en afirmar que tanto Magris como él son herejes y que Santo Tomás lo habría quema- 
do vivo, de haber oído sus ideas: "Tu come me sei un eretico, con tutto ciò che questo comporta per la Chiesa. San Tommaso ti avrebbe arso vivo; e dietro a lui tutti i gerarchi della Chiesa." (Magris; Marin 2014: 93)

Marin se convierte en un auténtico "consejero espiritual" para Magris en distintas ocasiones. En la carta del 8 de mayo de 1977, un Magris desconsolado ante la noticia de la aparición de un tumor cerebral en su sobrina de tan sólo cinco años de edad, confiesa al poeta que su fe se tambalea ante la injusticia y el absurdo de un dolor así. Marin da respuesta a esta desgarradora confesión, desde la experiencia personal de haber perdido al amado hijo con tan sólo 24 años. El poeta pide al amigo que no haga una lectura racional de la presencia del dolor en el caos del mundo, puesto que la incomprensión lo llevará a la desesperación. Antes bien, le invita a vivir en clave de gratitud; a no entender la vida como un derecho, sino como un deber y un don gratuito: "Il cosmo è frutto più di grazia che di conquista; e nessuno può dire: ne ho diritto! Non si ha neanche il diritto di vivere. Se mai un dovere. Siamo gìa nell'ambito dell'arroganza quando pretendiamo il bene, la vita." (Magris; Marin 2014: 338)

Dos grandes cuestiones reaparecen de manera recurrente en el diálogo epistolar sobre la divinidad: "el buen combate" paulino y el Panta rei platónico. Encontramos en la confesión del propio Magris a Sanson el calado de la enseñanza mariniana sobre el buen combate:

Lo direi con quella frase di Marin che ha veramente marcato il mio modo di essere: "le nostre contingenze colorano l'eternità di Dio". Ė anche per questo che io cito sempre Marin, il quale poi indirettamente mi ha molto aiutato anche in quel "buon combattimento" che certo appartiene a San Paolo, ma io ripeto, forse anche troppo spesso, abbinandolo a Biagio Marin. (Sanson [Magris; Marin 2014]: 390)

En cuanto al continuo devenir del mundo, el fluir imparable de la mutación del ser humano, la espiritualidad de nuestros dos protagonistas se acerca mucho a la intuición de ese otro gran poeta italiano del Siglo XX, Mario Luzi. Todo fluye, nada permanece, sólo Dios es inmutable; y la huella de esa inmutabilidad divina en la vida terrena la encontramos en la poesía, auténtica "gracia" que Dios concede a unos cuantos privilegiados: "Sì, la poesia è l'unico modo per raggiungere la vita eterna" (Magris; Marin 2014: 96).

\section{Conclusión}

Como hemos podido comprobar, la grandeza y la originalidad del epistolario de Claudio Magris y Biagio Marin está en que, en estas cartas, no sólo encontramos retazos de vida, sino lecciones de vida. Es el espíritu de estos dos grandes hombres de las letras italianas lo que encontramos en sus escritos, tal y como señala Sanson:

Marin considera la lettera come un genere a sé stante, in parte simile alla conversazione, in cui si misurano la vicinanza o la distanza tra gli uomini. Dove quel che 
importa non è la mera comunicazione a corrente alternata, bensì il passaggio dello spirito, l'instaurazione di un dialogo veramente "vitale", con la certezza per entrambi gli interlocutori di aver ottenuto -oltre che dato -molto di bene. (Motta 2013: 32)

Son muchas las referencias a la huella que el poeta de Grado ha dejado en el espíritu del gran germanista italiano. Es el propio Claudio Magris quien deja constancia de ello en numerosas ocasiones, no sólo en las cartas dirigidas a su maestro y padre espiritual, sino en entrevistas y ensayos publicados después de la muerte de Marin. Magris confiesa que Marin es una semilla en su interior que despierta la fuerza y la luz en él (Magris; Marin 2014: 285). Desde el inicio hasta el final de la relación, Magris se sintió el heredero espiritual y literario del poeta. Especialmente emotivas son las palabras que le dirige a su maestro el 20 de febrero de 1975, en las que le confiesa lo mucho que le debe y cómo, a pesar de las diferencias que aparentemente les separan, siempre ha intentado seguir sus pasos:

Ancora una volta ho ricevuto da te forza, chiarezza, armonia e salute. Mi sento il tuo erede, e sento la tua vigorosa luce trasfondersi in me, divenire una parte di me. [...] Io ti devo tanto. Naturalmente sono diverso da te, talvolta devo usare altri metri e altri linguaggi verso la vita, perché ogni uomo e ogni generazione devono tradurre l'eterno in forma e modi contingenti, a loro congeniali. Ma anche quando sembro lontano da te, è la tua via che cerco di battere -con passi miei. (Magris; Marin 2014: 316)

En el otro lado de la moneda descubrimos la mano de Magris en el reconocimiento internacional de la poesía de Biagio Marin. El propio Marin admitía el obstáculo que él mismo impuso a la difusión de sus poemas con el uso del dialecto graisan. Necesitaba un Juan Bautista que mostrara al mundo la grandeza de sus versos disfrazados de regionalismo, pero con una esencia universal. Finalmente encontró ese apóstol para su obra gracias al afecto, la admiración y la fidelidad del gran Claudio Magris.

El epistolario que ha llegado hasta nosotros es un válido instrumento de análisis para el lector o estudioso de la obra mariniana o magrisiana, puesto que en estas páginas llenas de vida, filosofía y poesía encontramos la esencia pura de estas dos grandes figuras de las letras italianas.

\section{BIBLIOGRAFÍA}

MAGRIS, Claudio (1992): Otro mar. Barcelona: Anagrama.

(1997 [1988]): Danubio. Barcelona: Anagrama.

(1999): Microcosmos. Barcelona: Anagrama.

(2008): El infinito viajar. Barcelona: Anagrama.

(2009 [1963]): Il mito asburgico nella letteratura austriaca moderna. Torino: Einaudi.

MAGRIS, Claudio; MARIN, Biagio (2014): Ti devo tanto di ciò che sono. Milano: Garzanti. 
MARIN, Biagio (1980): Nel silenzio più teso. (A cura di Edda Serra. Introduzione di Claudio Magris). Milano: Rizzoli.

(1991): Poesie. (A cura di Claudio Magris e Edda Serra). Milano: Garzanti.

MENGALDO, Vincenzo (1978): Poeti italiani del Novecento. Milano: Mondadori.

MOTTA, Antonio (2013): "Carteggio Magris-Marin "Caro Claudio... ", "Caro Marin... » Epistolario (1958-1985)". Il Giannone. Il tempo in fuga. Omaggio a Claudio Magris. Vol. 21: 27-52.

SANSON, Renzo (2014): "Canto e controcanto. Conversazione con Claudio Magris". Ti devo tanto di ciò che sono. Milano: Garzanti.

SERRA, Edda (1992): Biaggio Marin. Pordenone: Edizioni Studio Tesi.

\section{PERFIL ACADÉMICO Y PROFESIONAL}

Encarna Esteban Bernabé es Profesora de Lengua Italiana de la Universidad de Murcia. Licenciada en Filología Francesa (1999) y Doctora por la Universidad de Murcia (2015) tras la defensa de la tesis "La espiritualidad de Mario Luzi en su obra poética”.

Líneas de investigación: Filología Italiana, Didáctica de las segundas lenguas (Italiano y Francés); Inclusión de la Literatura en el aula de idiomas; Poesía italiana del Siglo XX.

Fecha de recepción: 15/05/2019

Fecha de aceptación: 08/07/2019 